\title{
Réhabilitation des héritages industriels et miniers et développement transfrontalier dans le bassin houiller sarro-lorrain
}

Mining and industrial heritage ad renewal of cross-border development in the coal basin in Sarre and Lorraine

Industriekulturelles Erbe und Wiederbelebung der grenzübergreifenden

Kooperation im saarländischen lothringischen Kohlenrevier

Juliette Ripp

\section{OpenEdition}

\section{Journals}

Édition électronique

URL : http://journals.openedition.org/rge/1436

DOI : $10.4000 /$ rge. 1436

ISSN : 2108-6478

Éditeur

Association des géographes de l'Est

Édition imprimée

Date de publication : 1 juin 2006

ISSN : 0035-3213

\section{Référence électronique}

Juliette Ripp, « Réhabilitation des héritages industriels et miniers et développement transfrontalier dans le bassin houiller sarro-lorrain », Revue Géographique de l'Est [En ligne], vol. 46 / 3-4 | 2006, mis en ligne le 18 décembre 2009, consulté le 08 septembre 2020. URL : http://journals.openedition.org/rge/ 1436 ; DOI : https://doi.org/10.4000/rge.1436

Ce document a été généré automatiquement le 8 septembre 2020.

Tous droits réservés 


\title{
Réhabilitation des héritages industriels et miniers et développement transfrontalier dans le bassin houiller sarro-lorrain
}

\author{
Mining and industrial heritage ad renewal of cross-border development in the \\ coal basin in Sarre and Lorraine \\ Industriekulturelles Erbe und Wiederbelebung der grenzübergreifenden \\ Kooperation im saarländischen lothringischen Kohlenrevier
}

Juliette Ripp

\section{Introduction}

1 Pilier de l'industrialisation de la Sarre et de la Lorraine, l'exploitation houillère a laissé en héritage des sites et des paysages, longtemps considérés comme les symboles d'une crise économique dont on a cherché à s'affranchir en supprimant des installations souvent d'une grande valeur architecturale, voire paysagère. De même, en raison de contraintes techniques et réglementaires liées aux risques environnementaux et sanitaires qu'elles représentaient, les friches minières ont été laissées en l'état, sans être ni valorisées ni réintégrées dans le tissu urbain des communes du bassin. Avec le retrait d'ici 2006 à 2010 des charbonnages français et sarrois, s'est engagée une prise de conscience collatérale en Sarre et en Lorraine visant à reconvertir ces espaces marqués par la monoindustrie en espaces porteurs de développement. Jusqu'en 1996, date de l'initiative "Eurozone Sarrebruck-Forbach-Nord", chaque pays pour des raisons de différences économiques (fiscalité) et techniques (normes de sécurité), a mis en place ses propres stratégies de reconversion. Mais aujourd'hui celles-ci ont montré clairement leurs limites, notamment face à la concurrence économique accrue au niveau européen. Depuis les Accords de Karlsruhe, l'occasion a été saisie de mener, à 
l'échelle du territoire de projet que constitue l'agglomération transfrontalière Sarrebruck-Moselle Est (labellisée « Eurosdistrict » par les gouvernements allemands et français), une véritable politique commune de valorisation des friches minières en misant sur leur potentiel économique, urbain, culturel et paysager. Cette volonté, que traduit la mise en place du programme transfrontalier du « Parc de Développement de la Vallée de la Rosselle », est l'objet d'étude du présent article.

Dans le cadre de la phase d'amorce du projet de «Parc de Développement de la Vallée de la Rosselle ", il se propose de dégager des potentiels pour tenter une même approche sur la zone Sarre-Moselle. Il présente six friches, qui, reliées entre elles par l'axe vert de la Rosselle, sont toutes porteuses de valeurs historiques, culturelles et paysagères fortes, ainsi que d'enjeux de recomposition économique et urbaine. Il conclut sur l'intérêt d'un programme d'aménagement à la fois transversal et qualitatif pour une valorisation durable de l'agglomération transfrontalière, en tant qu'Eurométropole.

\section{Le parc de développement de la vallée de la Rosselle : un projet de requalification de la « colonne vertébrale » du bassin houiller sarro-lorrain}

\section{A. Une prise de conscience ancienne mais cantonnée jusqu'alors aux frontières nationales}

3 La zone Sarre-Rosselle rassemble, autour du massif du Warndt, la plupart des anciens sièges d'exploitation de la houille et de l'industrie sidérurgique en Sarre et en Lorraine. Ces activités ont façonné un ensemble caractérisé par une urbanisation et une industrialisation massives, ayant presque entièrement comblé les fonds de vallée (figure 1).

Figure 1 : les emprises minières dans le nord-est du bassin houiller lorrain : une reconversion à vocation tertiaire et résidentielle

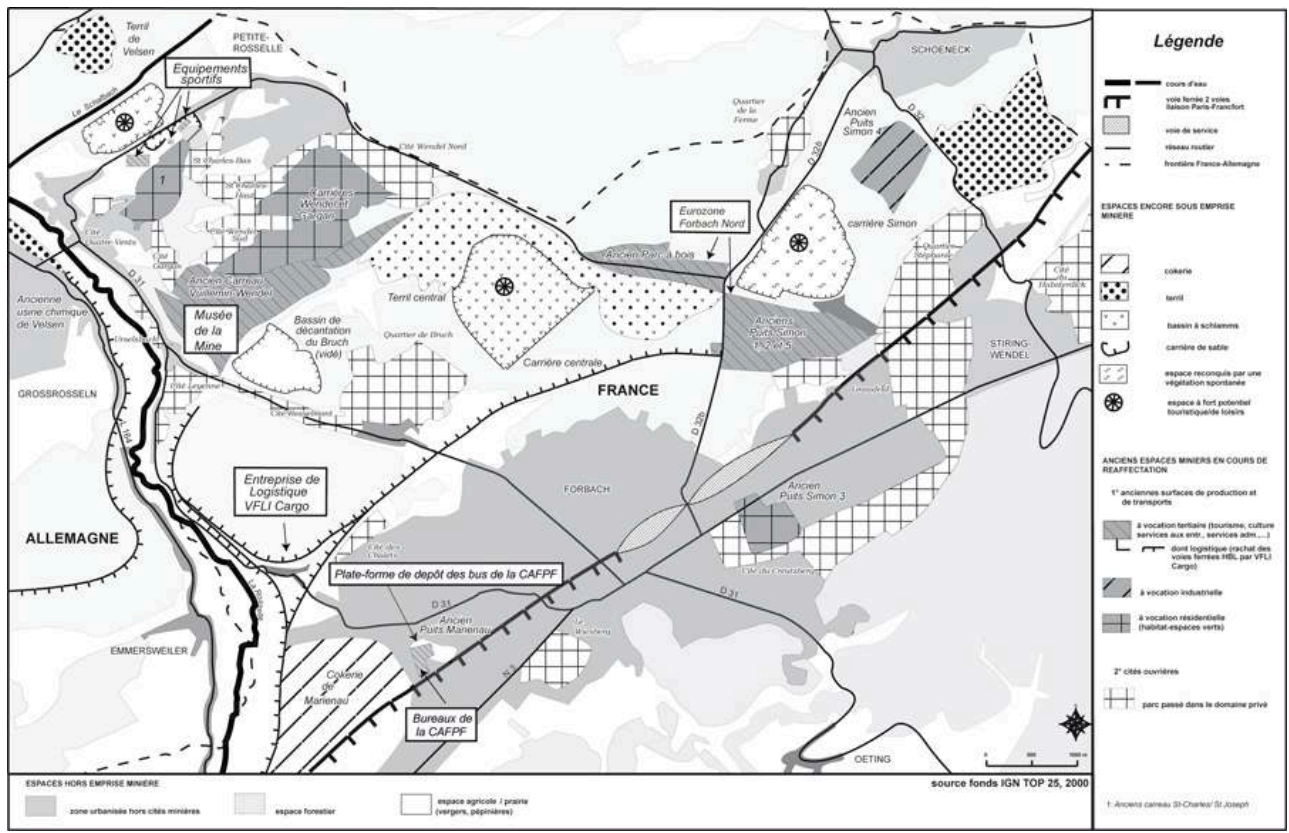


Dans cet ensemble, le cœur du bassin houiller lorrain longe la frontière allemande et présente un "paysage naturel» vallonné et fortement boisé. L'ensemble compose un riche patrimoine culturel, c'est-à-dire des espaces appropriés et remaniés par l'homme, témoins de l'évolution de l'utilisation des sols, dont il importe de conserver le caractère original.

5 La vallée de la Rosselle a fait l'objet déjà de multiples études. Elle a attiré l'attention du public du fait de son statut de rivière la plus polluée d'Europe, mais surtout en raison de sa position transfrontalière, la Rosselle prenant sa source en France. Les collectivités territoriales allemandes et françaises ont engagé depuis une vingtaine d'années une politique pour dépolluer la rivière qui a donné des résultats très satisfaisants, en témoigne le retour progressif de la flore et de la faune indigènes. Après les succès apportés par l'amélioration de la qualité de l'eau, l'obligation de réaménager les anciens sites miniers se prête aujourd'hui comme occasion pour engager un véritable développement durable de la vallée. La revalorisation paysagère de la vallée de la Rosselle est considérée comme faisant partie d'un projet global associant objectifs économiques, écologiques, urbains et culturels. La vallée, colonne vertébrale de l'ancien bassin houiller, représente aujourd'hui le lien majeur entre la France et l'Allemagne, entre les anciennes aciéries de Völklingen, classées au patrimoine mondial de l'Unesco, et le Musée du carreau Wendel, à Petite-Rosselle, points d'attraction majeur de la vallée. Toutefois, en raison de différences économiques (fiscalité) et techniques (normes de sécurité), chaque pays a jusqu'alors mis en place ses propres stratégies de reconversion. Celles-ci montrent aujourd'hui clairement leurs limites, notamment face à une concurrence économique toujours accrue au niveau européen. C'est pourquoi, les collectivités sarroises et lorraines cherchent depuis quelques années à surmonter leurs différences, en mettant en place des complémentarités en matière de développement territorial et de planification urbaine. Sans être directement opérationnelles, les pistes de réflexion concernant la vallée de la Rosselle jettent les premières bases d'une réflexion commune.

\section{B. Les acteurs}

6 Dans ce contexte, la Saarprojekt AG, entreprise affiliée à la RAG AG, la ville de Völklingen, le Land de Sarre, les communes du bassin houiller lorrain, la région Lorraine et l'Etat français ont lancé le projet du « Parc de Développement de la Vallée de la Rosselle». La ville de Völklingen a commandité une étude bilingue, la Machbachkeitsstudie: Das Zukunftsprogramm Rosseltal, (Étude de faisabilité: Parc de Développement de la Vallée de la Rosselle)'à la Gesellschaft für Innovations- und Unternhmensförderung (GIU) et à l'EPF Lorraine, afin d'analyser la faisabilité d'un ambitieux programme d'investissements transfrontaliers autour de la valorisation paysagère de la culture industrielle. Sur le plan politique, l'ensemble des collectivités locales potentiellement concernées ont été conviées à se joindre à la démarche en adoptant une résolution commune. 


\section{Une fédération d'énergies dans un cadre spatio-temporel strict pour des objectifs complémentaires et transversaux}

7 Le projet prévoit de concentrer les efforts sur la vallée de la Rosselle, espace de liaison thématique et géographique cohérent, à raison d'un investissement massif, annoncé à hauteur de 150 millions d'euros répartis sur 10 ans (2000-2010). Il s'agit donc d'une entreprise à long terme, appelée à drainer au maximum les intérêts et les volontés : milieux professionnels, politiques, investisseurs, mais aussi population et médias. Dans une vision large et constructive, on cherche à intégrer et à donner une nouvelle impulsion aux projets en cours, par l'« effet amplificateur de la labellisation» (parc d'activités « Eurozone », « Eurodistrict »).

8 La reconversion d'anciens sites industriels, la mise en lumière de dimensions culturelles et historiques porteuses d'identité, se trouvent au cœur d'un processus de mutation de grande ampleur, passant par :

- une recomposition paysagère des sites, garantes d'une haute qualité de composition, évitant un simple « verdissement » et valorisant le potentiel écologique ;

- une mise en sécurité des espaces délaissés par le traitement du risque de contamination ;

- une mise en scène de l'« échelle de la monumentalité », faisant des traces de l'activité industrielle des « points d'accroche » dans le paysage, visibles sur de longues distances et révélant la dimension artistiques des lieux, leur singularité et leur rareté ;

- une amélioration de la structure urbaine, par la mise en relation des sites, des centres-villes et des espaces naturels (bois et berges de cours d'eau par exemple);

- une priorité au recyclage des friches, pour limiter l'urbanisation et mettre en valeur les espaces libres, selon un principe de mixité des fonctions urbaines.

Toutes ces interventions devront concourir à construire une image positive et dynamisante du territoire, indispensable à l'attraction de nouvelles activités et de services innovants, avec à la clé, la reconquête d'une position économique forte.

Il ne s'agit pas dans notre étude, d'étudier toutes les composantes de ce vaste programme les unes après les autres, mais de partir de l'analyse d'un certain nombre de friches minières (et sidérurgiques), pour déterminer, en raison de la complémentarité de leur vocation et de leur potentiel (carreau, terrils, carrières, usines, bassins de décantation), en quoi un certain nombre d'objectifs transversaux du programme peuvent être atteints.

\section{Des friches aux potentiels variés à valoriser dans une approche globale et transfrontalière}

11 L'approche suivie ici a tenté de se caler au mieux dans ce contexte particulier d'amorce de coopération. Sans rechercher l'exhaustivité, elle privilégie la diversité des situations, tant dans le contexte local, l'état d'avancement des projets que dans les problématiques en jeu, au travers de cinq études de cas. 


\section{A. Le Carreau Wendel/Commune de Petite-Rosselle : témoin de l'âge d'or industriel d'après-guerre}

Reconverti en partie en musée de la mine, le site Wendel jouit d'une place privilégiée pour un rapprochement touristique et culturel franco-allemand (photo 1). Cet ancien site d'extraction et de traitement du charbon d'environ 60 ha, situé en plein cœur du bassin houiller, constitue l'un des témoins les plus impressionnants de l'industrie lourde des $\mathrm{XIX}^{\mathrm{e}}$ et $\mathrm{XX}^{\mathrm{e}}$ siècle (1866/1986), ayant structuré l'ensemble du paysage environnant (présence des chevalements, lavoirs, décanteurs, magasins, réseau de voies ferrées, extensions urbaines sous forme de cités, creusement de la carrière et formation du terril,...). Au delà de l'intérêt lié au caractère monumental du site, on retient l'intérêt patrimonial que présente la diversité des architectures marquant les différentes logiques industrielles qui se sont superposées sur le carreau; les extensions d'après-guerre sont directement lisibles par l'importance et l'homogénéité des volumes simples et rigoureux de fer et de briques. En témoigne la structure du lavoir, directement visible depuis la route d'accès, qui est une sorte de collage d'architectures aux formes massives et complexes ayant connu neuf extensions importantes de 1891 à 1970 .

Photo 1 : le Carreau Wendel vu en direction du sud depuis le rebord de la carrière

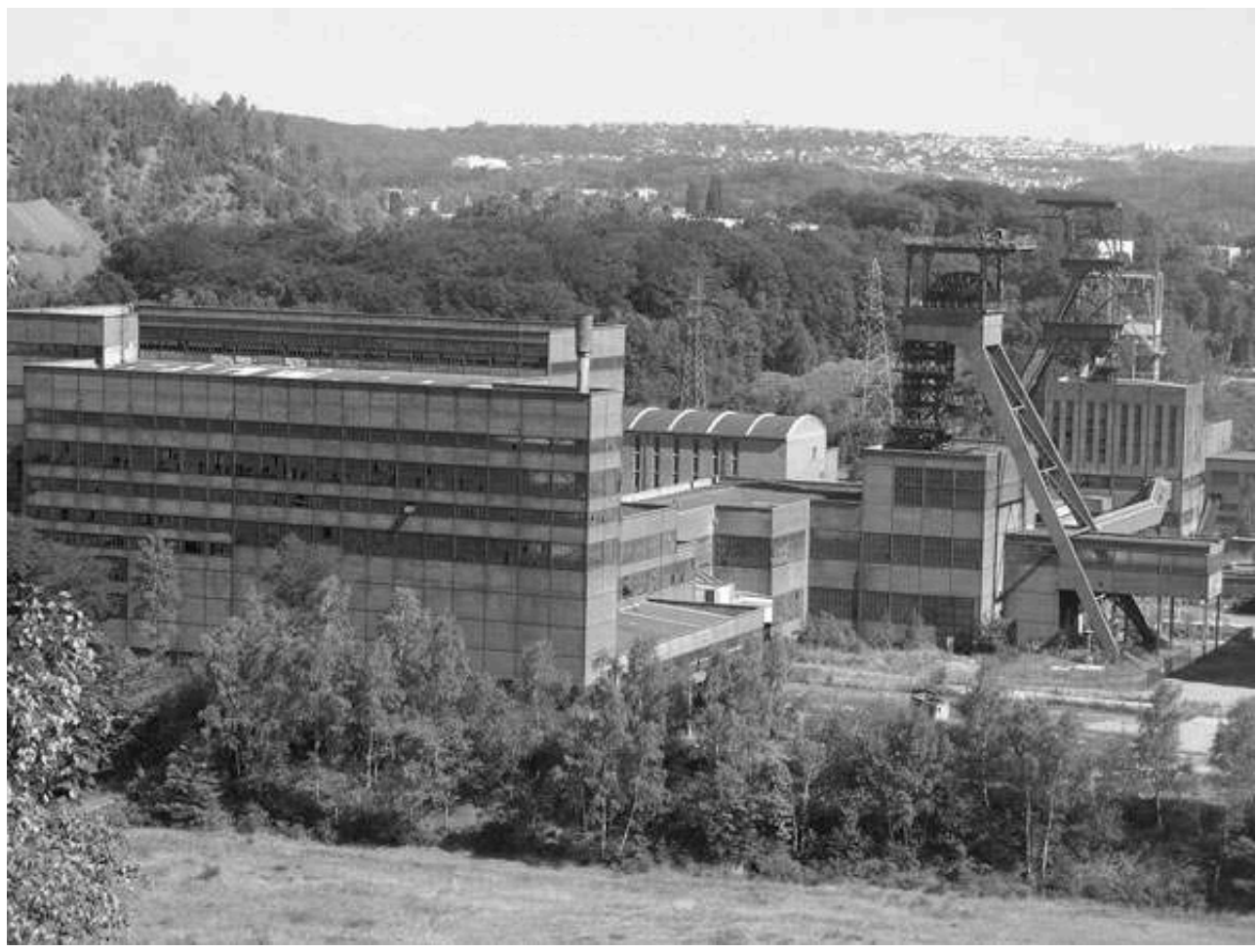

Avec ses trois chevalements et ses lavoirs il constitue un témoignage exceptionnel de l'évolution de l'architecture industrielle au cours du XXè siècle. A l'arrière-plan on aperçoit la côte constituant le rebord du Warndt.

photo M. Deshaies, 2006 
Photo 2 : le nouveau musée de l'histoire industrielle du Carreau Wendel et le terril encore partiellement végétalisé photo M. Deshaies, 2006

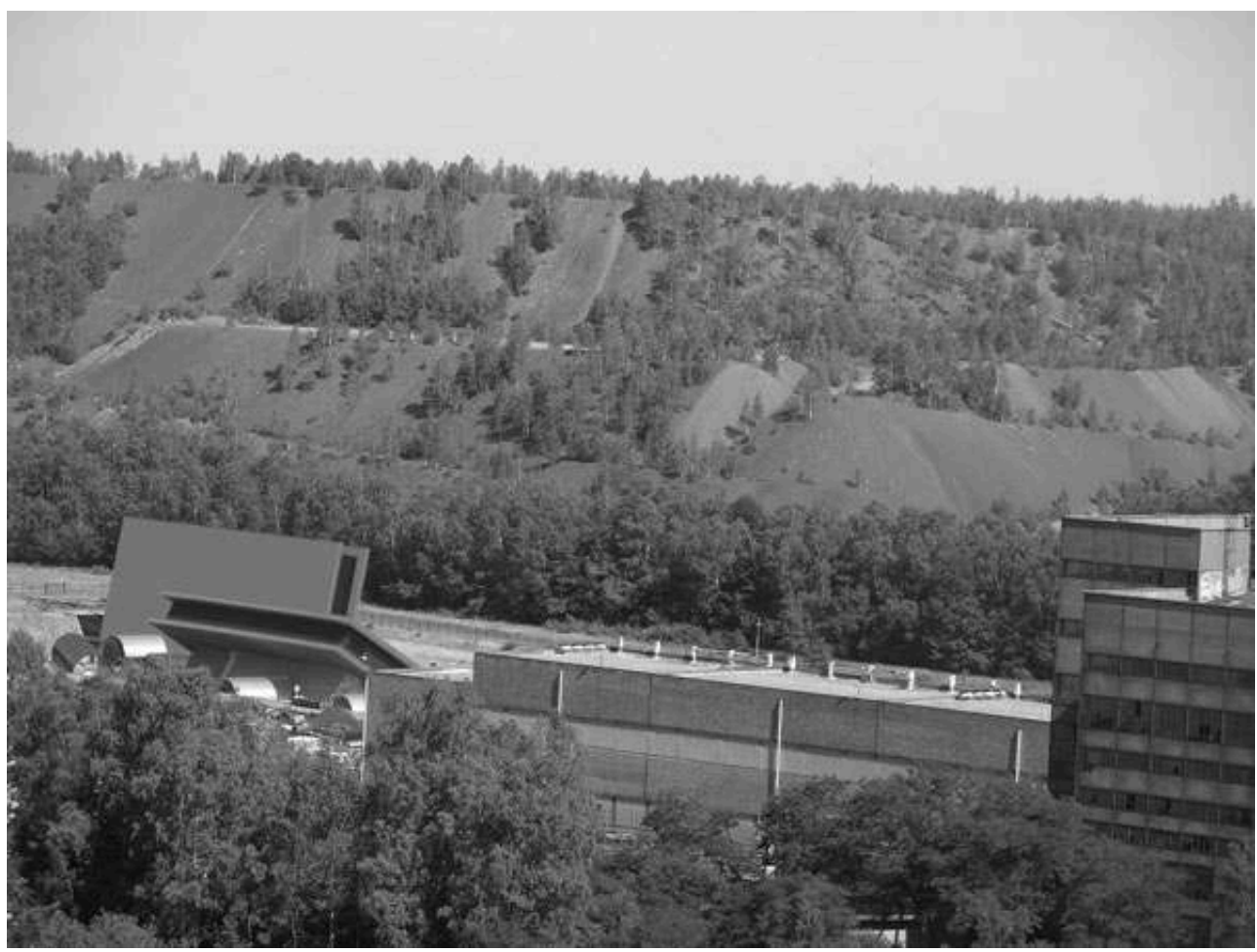

photo M. Deshaies, 2006

Depuis 1988, les acteurs, le Centre de Culture Scientifique et Technique Industrielle (CCSTI) puis le Syndicat Mixte pour la création et la gestion du Musée de la mine, né du rapprochement de la Communauté d'Agglomération de Forbach et de la Communauté de Communes de Freyming-Merlebach, l'EPF Lorraine, la région Lorraine, l'Union Européenne, le Ministère de la Culture et les HBL, se sont associés afin de redonner vie à ce lieu d'une grande valeur historique et paysagère en menant une requalification du site sur le long terme, privilégiant trois axes :

- la réalisation, pour 2007, d'un lieu de mémoire de l'histoire industrielle et sociale locale, préservant le patrimoine en place et exploitant les qualités pédagogiques du site pour l'ouvrir aux visiteurs : circuit du mineur avant sa descente au fond (salle des pendus, infirmerie, lampisterie,...), réalisation en surface d'une reconstitution des chantiers de fond à ciel ouvert (photo 2), exposition sur le charbon, les techniques de la mine, la vie du mineur, etc.;

- l'animation et la rentabilisation des investissements réalisés par l'organisation d'une vie culturelle (spectacles, expositions temporaires complémentaires), en collaboration avec le Théâtre « Carreau, scène nationale de Forbach »;

- le développement de la culture scientifique par la création d'un pôle de conférences, centre de recherche et de documentation sur les innovations techniques (énergie, sous-sol, pollution, chimie), et l'histoire industrielle (ethnographie, archives industrielles) en vue de créer un vivier de nouvelles pistes pour la reconversion du bassin.

14 À terme, il s'agit de passer d'une structure touristique d'envergure locale à une structure d'envergure régionale (le Musée a d'ores et déjà été classé « Musée d'État »), à l'échelle sarro-lorraine, en tissant des liens durables avec le musée des aciéries de Völklingen («Weltkulturerbe Völklinger Hütte»). Actuellement, les acteurs travaillent 
à l'élaboration d'un concept de communication commun, dont la diffusion sera assurée par un bureau de coordination franco-allemand. Au programme : expositions couplées, tarifications communes, et produits clés en main (brochures bilingues avec informations concernant l'hébergement, la restauration et les curiosités dans la région, les horaires d'ouvertures, organisation de navettes spéciales en bus entre les deux sites). Le tourisme industriel étant en plein développement en Moselle et en Sarre, suite à la fermeture des dernières unités d'exploitation, ces deux sites frontaliers devraient, grâce à une coopération renforcée, attirer de nombreux touristes à l'échelle frontalière comme européenne.

Depuis 1991, L'EPFL a été sollicité, en vertu de la politique régionale de TED², pour intervenir sur l'ensemble du site, afin de réaliser les travaux d'aménagement préalables à l'installation du musée, tels que le démantèlement des rampes ferroviaires, le préverdissement $\mathrm{du}$ site, la réalisation d'une route d'accès est/ouest, la réfection du clos et couvert de certains bâtiments ainsi que le classement de ceux-ci à l'Inventaire Supplémentaire des Monuments Historiques.

En vue de favoriser le développement urbain de la commune de Petite-Rosselle dans une optique de mixité des fonctions urbaines, une partie de l'emprise du musée est destinée à l'accueil d'une aire d'extension mixte (activités, logements), à intégrer de façon harmonieuse au site du musée (chemins de promenades et espaces verts). Conçus dans un souci de cohésion urbaine, ces aménagements constitueront un moyen original de faire du musée un lieu de transition entre les nouveaux quartiers est, et ceux au Nord, la cité Wendel sud.

\section{B. L'Usine Sidérurgique de Völklingen, Patrimoine Mondial de l'Unesco}

Classée au patrimoine mondial de l'Unesco en 1994, l'usine sidérurgique de Völklingen possède des installations exceptionnellement conservées et compactes, illustrant l'histoire industrielle et économique de la région (photo 3). L'un des objectifs, à terme, du « Parc de Développement de la Vallée de la Rosselle " (figure 2) est de donner corps à un arc entre les deux « projets-phare » que sont l'usine de Völklingen et le Musée du carreau Wendel, ponctué par le développement d'autres sites décentralisés (carreau de Velsen, carreaux St-Charles/St-Joseph, carreau du Warndt en Sarre, forêt et moulin du Guensbach...) 
Figure 2 : l'espace de projet transfrontalier « Parc de développement de la vallée de la Rosselle »

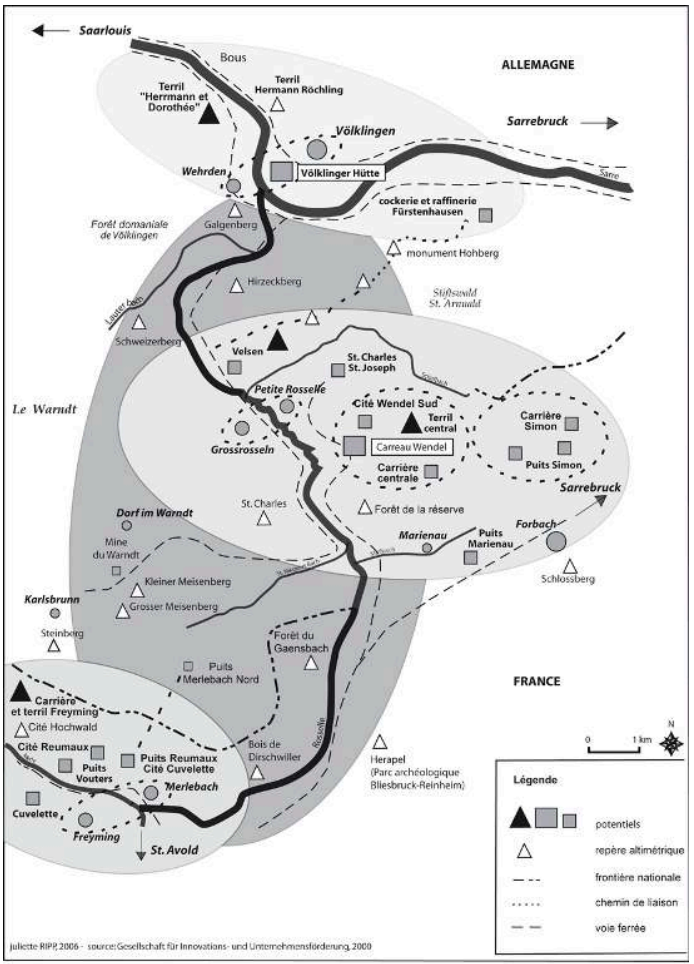

Photo 3 : l'ancienne usine sidérurgique de Völklingen

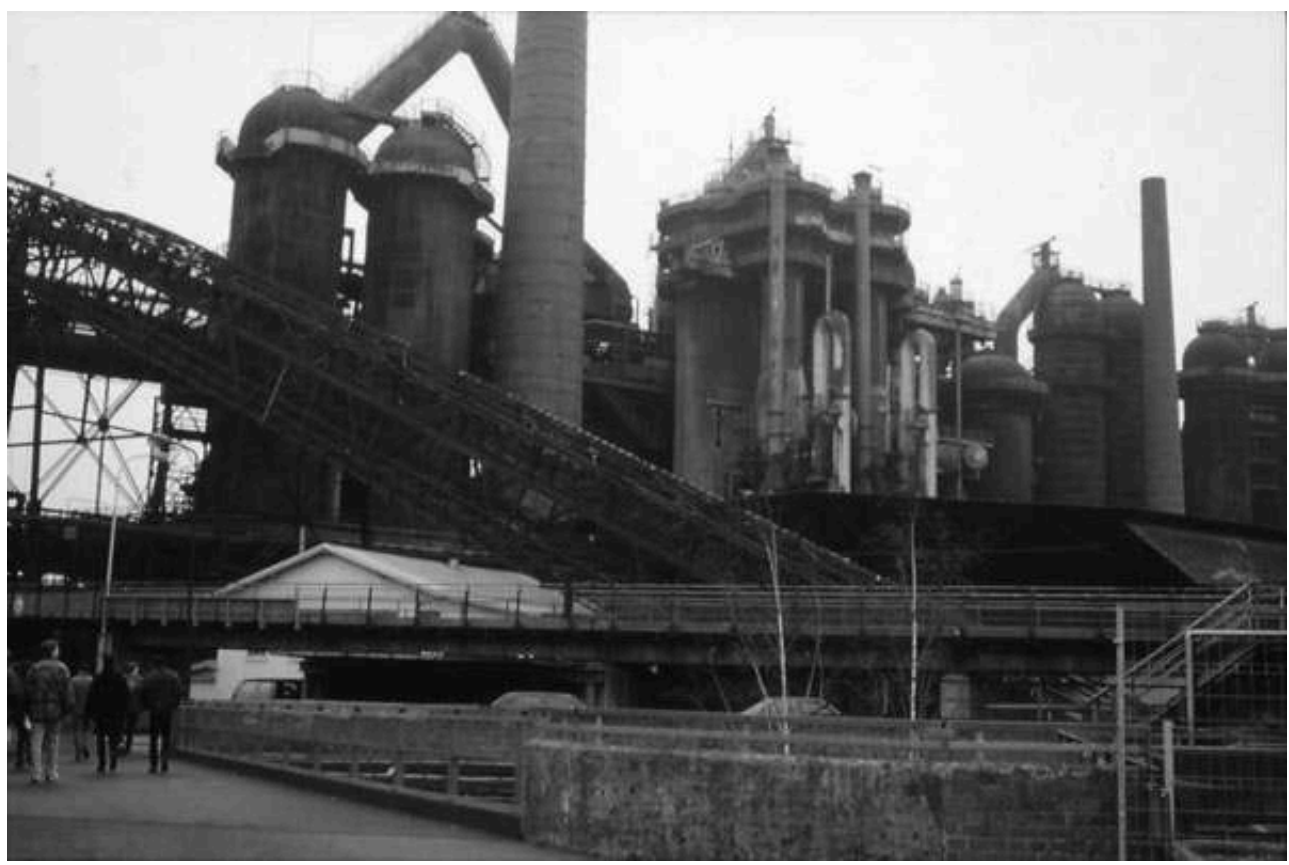

Fermée en 1986, I'usine a été classée au Patrimoine mondial de l'Unesco en 1994.

photo M. Deshaies, 1999

18 Ce complexe sidérurgique d'environ 60 ha, en activité de 1881 à 1986, est constitué d'un ensemble d'espaces et de décors industriels gigantesques, témoins de l'évolution des technologies de pointes et notamment des grandes réalisations technologiques du 
début du XXe (batteries de 6 hauts-fourneaux de $45 \mathrm{~m}$ sur $250 \mathrm{~m}$, cokerie avec ses 104 fours à coke, plateformes des chaudières, salle des souffleries de $6000 \mathrm{~m}^{2}$, château d'eau,...). Acquis en 1881 par Hermann Röchling, elle est considérée à l'époque comme l'usine sidérurgique la plus innovante et la plus productive d'Europe : l'installation de frittage est la plus grande de son type au monde, tandis que le château d'eau central de 1918 est le premier bâtiment construit sur pilier en béton en Europe. Monument industriel « icône » de la prospérité économique de l'Allemagne d'Après-guerre, dans le contexte de la CECA, elle est aussi le symbole visible du centre économique de l'Europe actuelle.

19 En 1999, le Land de Sarre ainsi que la ville de Völklingen, propriétaires du site, ont créé l'association "Weltkulturerbe Völklinger Hütte», chargée de développer un programme de réaménagement de l'usine sidérurgique en musée et en centre interactif de la culture industrielle (aménagement des locaux pour l'accueil de visiteurs, de passerelles et de balustrades longeant les installations extérieures, production de matériel d'information, documents de vulgarisation du travail de recherche réalisé sur l'histoire de l'usine et les procédés techniques de production, brochures, tickets, site internet).

Par ailleurs, l'usine disposant d'une situation privilégiée sur un îlot en rive gauche de la Sarre au centre-ville de Völklingen, il est actuellement question de l'insérer au cœur d'un projet de réaménagement urbain du quartier « défraîchi » de la City, dont il doit contribuer à stimuler l'attractivité. Il s'agit ainsi de faire du musée un espace non plus fermé mais ouvert, dont la fonction doit être d'assurer une liaison verte entre les quartiers de la City et de Wehrden, et d'ouvrir le centre-ville sur la Sarre (mise en scène de «la ville au bord du fleuve »). Par ailleurs, les emprises de l'usine et de l'îlot qui ne seront pas intégrées au musée, devraient participer à la diversification de l'économie urbaine et accueillir commerces de détail, services aux entreprises, activités artisanales, annexe de l'université des beaux-arts de Sarrebruck, Le Centre de technologies,...).

21 Enfin, à terme, il est prévu de reconstituer l'unité paysagère que formaient l'usine et ses terrils (au nord-ouest de l'usine), dont l'originalité réside dans leur forme conique. Pour lire et retrouver un sens à cette unité paysagère, alliance subtile de minéral, de végétal, et de formes géométriques pharaoniques, l'observateur doit pouvoir prendre $\mathrm{du}$ recul et de la hauteur, tirer partie de la topographie existante en ouvrant des perspectives plongeantes sur les coulisses de l'industrie de la vallée de la Sarre (usine de Völklingen, centrale électrique de Wehrden et ses imposante tours de refroidissement) à partir d'un point de vue panoramique (belvédère, illumination,...) mis en place sur les terrils.

\section{Le carreau de Velsen/périmètre de la ville de Sarrebruck : l'exploitation charbonnière au temps de l'administration prussienne}

22 Le secteur de la mine de Velsen constitue le point central du projet «Parc de Développement de la Vallée de la Rosselle " pour sa valeur paysagère : le lit majeur qui s'étend à l'est de la mine est constitué de prairies, dont la protection a fait l'objet d'un décret du Land de Sarre dès 1992. Le paysage magnifique qui borde les installations minières, est le premier élément qu'aperçoit le visiteur lorsqu'il a dépassé la confluence de la Rosselle et du Schafbach. L'urbanisation y est interdite depuis 1992 et des travaux 
de renaturation et de remblaiement avec du matériau de mine afin de lutter contre les inondations, $\mathrm{y}$ ont déjà été en partie réalisés. Cet ancien site d'extraction et de traitement du charbon, d'une surface d'environ 30 ha (1904-1965), présente des bâtiments d'architecture typiquement prussienne (pierres de taille en grès bigarré de la région, fenêtres à meneaux en plein cintre, perrons élégants et boucliers et armoiries, datant du début du XXe siècle (1904-1917), dont l'imposante homogénéité visuelle est à mettre en valeur, en contraste par exemple avec les bâtiments du carreau Wendel, qui pour la plupart témoignent de la logique industrielle d'Après-guerre. La machine d'extraction de la firme Dillinger, datant de 1916, est l'une des plus anciennes machines à vapeur en l'état du secteur sarrois et à ce titre, constitue une curiosité supplémentaire du site, d'autant plus qu'elle fonctionne encore, alimentée par la centrale thermique située à proximité.

23 Actuellement, le projet d'ouverture aux visiteurs du site avec ses installations industrielles bénéficie de la garantie de la participation financière et technique de trois acteurs : les DSK AG, le Land de Sarre ainsi que la ville de Sarrebruck. Il s'agit d'une part de recruter un conservateur et de réaliser un important travail d'archives puis de vulgariser les données pour les rendre accessibles aux visiteurs, de développer un concept de promotion du site et de commercialisation du musée (parcours de visite, possible utilisation de l'ancien petit train d'accès à la mine, aménagement des Schlafhäuser, dortoirs datant de 1912, matériel d'information,...). Il serait même intéressant d'employer d'anciens ouvriers des DSK, voire faire participer les apprentis évoluant encore sur le site dans une galerie d'apprentissage artificielle -, au concept de développement du musée, et de faire profiter les visiteurs de leur expérience de la mine (visites guidées,...).

24 Au niveau paysager, l'aspect pittoresque du site, façonné par les affaissements miniers est à intégrer dans un plan de cheminements transfrontaliers, par l'aménagement sur les crêtes de sentiers sécurisés de promenade. La présence de l'immense terril au nordouest de la mine ajoute au caractère pittoresque du paysage, puisqu'on en obtient un excellent point de vue sur l'ensemble de la vallée de la Rosselle et du Schaffbach, le bassin à schlamms, en France au puits St-Charles, et sur la ville de Petite-Rosselle. Un concept intégrant les installations industrielles, la mise en valeur des prairies, et le façonnement du terril est actuellement en cours d'élaboration par la GIU. De même, afin de soigner les franges du site, des aménagements paysagers plus précis seront réalisés sur le crassier de l'ancienne usine chimique, au sud-ouest de la mine : il s'agit de façonner des «jardins de l'industrie " sur lesquels une végétation de bouleaux aux troncs blancs et au feuillage vert offrira un contraste saisissant avec leur terreau.

\section{Le bassin de décantation des puits Saint-Charles/Saint-Joseph : du bassin à schlamms à la zone de loisirs transfrontalière}

Ce bassin à schlamms, d'une grande qualité paysagère, est appelé à devenir une vaste zone de loisirs, drainant un public en provenance de toute l'agglomération transfrontalière. La dimension du site, impressionnante, ne se mesure véritablement que sur les cartes et les photos aériennes. Le caractère pittoresque du site rempli de schlamms noirs, très fertiles, est renforcé par le contraste que génère l'existence d'une végétation spontanée. Un parcours a déjà été aménagé dans le vallon du Schaffbach, de même qu'un sentier, qui longe l'ensemble du bassin et ses franges boisées. Au sud, les 
fronts de taille des anciennes carrières, dont l'un recoupe l'espace en deux plateaux, découvrent un secteur boisé intéressant.

Le projet d'aménagement de la vallée du Schaffbach en zone transfrontalière de loisirs nautiques et sportifs, a fait l'objet d'études depuis le début des années 1970, actualisées au milieu des années 1990, sans donner lieu à aucune stratégie opérationnelle. Son potentiel de valorisation touristique pourrait toutefois donner lieu à des interventions envisagées en trois temps. Dans un premier temps: la maîtrise foncière publique des terrains situés de part et d'autre du ruisseau devrait être assurée, sachant que les terrains appartiennent essentiellement aux HBL et à la DSK. Des travaux de curage du bassin à schlamms après l'exploitation des schlamms par la SNET (Société exploitant la centrale E. Huchet de Carling) jusqu'en 2014 permettraient de l'étendre à une longueur de $500 \mathrm{~m}$. La deuxième phase consisterait à créer des sentiers forestiers, à agrandir l'accès actuel à la vallée, et à construire des locaux adaptés aux activités de plein air (voile, plongée, baignades), enfin à réaliser des aménagements paysagers. Le site du bassin, après exploitation par la SNET pourrait offrir des possibilités de passage pour une liaison piétonne et cyclable transfrontalière vers le site Wendel, dans la continuité des chemins réalisés en Sarre le long de la Rosselle. La troisième phase permettrait de diversifier et "fidéliser " la population concernée par la zone de loisirs grâce à la construction d'une voie de jonction entre la route d'accès inférieure et la route aménagée en seconde tranche, et l'aménagement d'un terrain de camping et d'une auberge de jeunesse.

\section{E. La zone d'activités transfrontalière « Eurozone Sarrebruck- Forbach Nord » : de l'ancienne friche minière à la technopole transfrontalière}

Intégré à part entière dans le projet du «Parc de Développement de la Vallée de la Rosselle " (figure 2), en raison de la qualité patrimoniale de ses bâtiments, le secteur des anciens puits Simon I, II et V, à Forbach et du quartier de la Brême d'Or à Sarrebruck est avant tout au cœur des enjeux économiques de la future agglomération transfrontalière, puisque cet espace est voué à l'accueil de la première technopole transfrontalière entre la France et l'Allemagne. 2003 est l'année du lancement à proprement parler de l'Eurozone puisqu'une première entreprise, Pemtech (usinage de haute technologie) s'est installée sur le site du parc à bois dans la partie française de cette Zone d'Aménagement Concertée. Grâce aux perspectives offertes par les Accords de Karlsruhe, il est désormais possible pour les collectivités locales porteuses de ce projet, à savoir la Communauté d'Agglomération de Forbach, assistée de l'EPF Lorraine au titre de la politique du TED ${ }^{3}$, du Conseil Général de la Moselle, de la ville de Sarrebruck et du Land de Sarre, de porter ensemble, par le biais d'une maîtrise d'ouvrage commune, une opération d'aménagement de grande envergure. C'est en effet essentiellement à travers le développement des compétences d'une technopole, telles que l'accueil de services publics et privés (télécommunications, poste), l'assistance aux créateurs (formation, recherche, management, assistance commerciale, " eurocompétences »), le développement d'outils de communication et de promotion économique, que l'agglomération transfrontalière pourra conforter sa qualité de "Métropole Européenne », en retrouvant progressivement son pouvoir de décision économique. 

d'environ 110 ha, est composée des plateformes hautes des puits Simon I, II, du carreau du puits Simon $V$ ainsi que du parc à bois, côté français, et du côté allemand, d'un ensemble composite de sites, les logements des anciens jardiniers du cimetière "Hauptfriedhof », du parking de ce dernier, ainsi que de l'ancien poste frontière et ses jardins. La qualité paysagère et patrimoniale des sites et des bâtiments en font des lieux attractifs pour l'implantation de sociétés soucieuses du cadre dans lequel elles évoluent. Sur le site Simon, la plupart des bâtiments sont classés à l'ISMH (la centrale des machines, notamment, construite par choret, architecte officiel de la famille Wendel, rappelle la gare d'Orsay), tandis qu' à Sarrebruck, les logements et jardins du cimetière, datant de 1933, sont classés au «Denkmalschutz ». Le relief accidenté des sites Simon peut par ailleurs devenir un atout, si l'on prend la mesure de l'impression visuelle de vallonnement, que renforce le creusement des trois carrières à proximité (photo 4). Le paysage de bois et de forêts qui inonde alentour le site le valorise du point de vue de l'attraction exercée sur les futures entreprises candidates. Les jardins du cimetière et de la douane, côté allemand, constituent des espaces verts interstitiels d'une grande diversité écologique (conifères, mélèzes, thuyas, rosiers sauvages, tilleuls, mûriers...) au sein même de la zone d'aménagement.

Photo 4 : Carrière, terrils et cadre forestier sur la bordure nord de Forbach

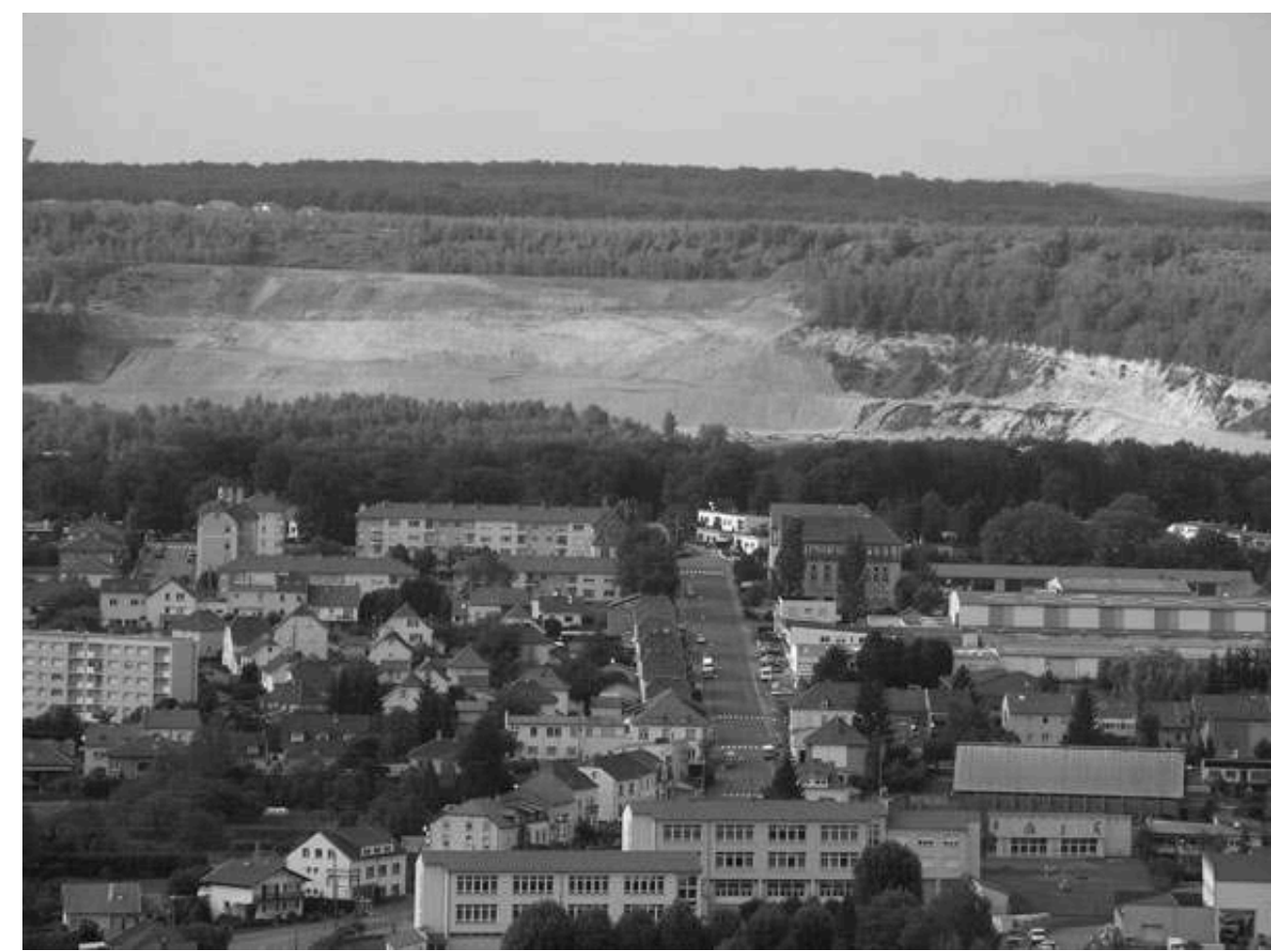

La carrière centrale du puits Simon en voie de réhabilitation. Après achèvement de la réhabilitation, ces anciennes friches minières constitueront des espaces verts attractifs au sein d'une agglomération très dense.

photo M. Deshaies, 2006

L'objectif principal de cet «outil» de développement économique est d'accueillir des activités industrielles de haute technologie et des activités de services, des " eurocompétences», adaptées à la demande d'investisseurs étrangers : conseil fiscal, 
juridique et économique, experts comptable, bureaux de traduction, centre de congrès, centres de formation interculturels (université franco-allemande), centre de formation en ligne. Il s'agit, dans le contexte européen, de permettre aux entreprises intéressées par le marché voisin de surmonter aisément les difficultés existantes de par la situation frontalière: différences de langage, de mentalités, d'organisation administrative et politique, de législation, de modes de distribution et techniques de commercialisation.

L'élaboration du projet vise à satisfaire quatre types de besoin :

- une organisation communautaire dans laquelle les entreprises sont proches (petites et moyennes entreprises) et partagent des services en commun : le « village d'entreprises » sur la plate-forme des puits I et II, le site de la douane et des logements des jardiniers à la Brême d'Or. Cette conception s'exprime dans l'espace, par la réalisation de petits bâtiments de 1000 à $2000 \mathrm{~m}^{2}$, peu distants les uns des autres, sans clôture et avec du parking en commun. Ce type d'organisation a l'avantage de permettre la reconversion des bâtiments existants des houillères, d'utiliser les petits bâtiments des jardiniers et de la douane (la surface des terrains nus est elle-même réduite) et de constituer un tout architectural cohérent, entre les bâtiments neufs et anciens. Il a l'avantage également de permettre l'éclosion d'une certaine convivialité : cheminements piétons, développement des espaces verts, et mise en valeur des jardins, réalisation de services communs ;

- une organisation plus classique ou chaque entreprise vit de façon relativement indépendante sur le parc à bois, et le puits Simon V : « une entreprise, une parcelle »; des clôtures végétales seront créées, qui viendront renforcer l'image recherchée de parc paysager. Ce parti pris d'aménagement sur ces sites provient du fait que les installations du puits $\mathrm{V}$ seront rasées, et que le parc à bois, complètement nu, libère une surface totale d'environ 50 ha ;

- une infrastructure de communication à haut débit accessible à des tarifs préférentiels sur l'ensemble de la zone, en France comme en Allemagne : la boucle NTIC, en cours d'élaboration par le Conseil Général de la Moselle, reliera toutes les communes du département en France. Le Land de Sarre compte investir dans la création d'un prolongement à partir de la frontière jusqu'à la Brême d'Or ;

- une amélioration des possibilités d'accès par la création d'une voie de désenclavement : liée d'une part à la nécessité de relier les deux « Europarcs », et d'autre part, de désengorger la RN3 qui draine plus de $40 \%$ des déplacements transfrontaliers vers Sarrebruck. Sur décision du Comité Régional d'Aménagement du Territoire, l'EPF Lorraine s'est vu attribuer la maîtrise d'ouvrage de cette infrastructure sur la partie française $(4,5 \mathrm{~km})$, et en Sarre, c'est le Landesbetrieb für Strassenbau des Saarlandes (service des Ponts et Chaussée du Land de Sarre), la Deutsche Bahn (qui réalisera un pont pour le passage de la voie au dessus de ses propres voies ferrées) et la GIU qui assurent la maitrise d'ouvrage d'une infrastructure de $810 \mathrm{~m}$. Actuellement, le projet en est au stade des études préliminaires (études d'impact, enquête publique) et de l'acquisition des terrains nécessaires.

31 À terme, l'aménagement et l'exploitation de l'Eurozone devraient constituer un ressort essentiel pour le développement urbain des deux villes. En effet, le raccordement des Europarcs de la Brême d'Or et de Forbach-Nord équipera les deux villes d'un nouvel axe routier. Ces aménagements permettront de faire face aux nouveaux transports générés par les activités économiques du secteur, tout en améliorant les capacités routières du tissu urbain commun. La création de l'Eurozone illustre à plus grande échelle la volonté de considérer un nouvel espace urbain commun, légitimant la nécessité d'élaborer un schéma de développement pour l'ensemble de l'agglomération franco-allemande. A 
plus long terme, les conséquences du développement d'un tel espace économique et les retombées pour la politique d'urbanisme devraient être l'illustration concrète d'une intégration européenne. Pour la Communauté d'Agglomération de Forbach notamment, qui a souffert d'un enclavement économique occasionné par une situation frontalière peu avantageuse avant les Accords de Schengen et les Accords de Karlsruhe, cette perspective est synonyme non seulement d'emplois mais aussi d'un développement durable dans un contexte franco-allemand.

\section{F. La requalification des berges de la Rosselle : I'axe de liaison des projets du «Parc de Développement de la Vallée de la Rosselle »}

L'urbanisation et l'exploitation minière sont à l'origine de nombreux aménagements sur son cours, et de nombreuses surfaces ont été rendues imperméables, altérant progressivement le lit majeur. Il faut noter que l'exploitation charbonnière (à Velsen, on a exploité jusqu'à $800 \mathrm{~m}$ de profondeur) a généré un affaissement de la vallée atteignant parfois douze mètres et une aggravation des risques d'inondation du lit majeur lors des pics de crues. En 1991, les SBW AG, conformément à leurs obligations d'exploitant houiller et à une étude réalisée en 1987 par le Stadtverband de Sarrebruck (Björnsen AG Studie), ont procédé au remblaiement de certaines parties de la prairie avec des matériaux de mines, jugeant cette action plus efficace que la construction de nouvelles digues de protection. L'étude prévoyait une autre action, à entreprendre ultérieurement pour renforcer le dispositif: reconstituer dans le lit majeur de la Rosselle des espaces d'expansion des crues, tout en prenant des mesures de protection de la faune et de la flore ${ }^{4}$. En Lorraine, il a également été prévu, mais plus tardivement, de recréer un lit majeur fonctionnel apte à favoriser l'expansion des crues, conformément aux préconisations du Plan de Prévention des Risques d'Inondation prescrit en décembre 2001 (l'ensemble des aménagements permettra un gain de surface inondable de $24000 \mathrm{~m}^{2}$ ) par Arrêté du Préfet de la Moselle.

La concordance des objectifs en Moselle et en Sarre a progressivement amené les acteurs de part et d'autre de la frontière, réunis désormais au sein de l'association 
ZSMA, à réfléchir à une opération de grande envergure pour la renaturation et la requalification des berges de la Rosselle sur l'ensemble de son cours. Au niveau de la qualité paysagère, il est prévu de :

- redévelopper la ripisylve, qui, quand elle n'a pas disparu à certains endroits, est peu diversifiée et vieillissante, et altère la capacité d'autoépuration de la rivière ;

- permettre une diversification des habitats et des espèces (création de zones humides, remise en eau de bras morts, nouvelles plantations, protection des pieds de berges, pose de géotextile, débroussaillage sélectif).

Enfin, sur le plan socio-économique, le projet devrait avoir des impacts positifs puisqu'il constitue un facteur certain de revalorisation pour les communes traversées. Il est prévu de créer des connexions avec les autres ressources touristiques du site (autres chemins de randonnée, pistes VTT, accès par le cheminement aux différents musées et curiosités, carreaux de mines, éléments marquants du paysage minier comme les carrières et les terrils.) et de permettre ainsi à la population locale de se réapproprier l'espace environnant, la rive longtemps confisquée par l'industrie minière.

\section{Vers une dynamique de « parc de développement de la vallée de la Rosselle »}

\section{A. Synthèse des études de cas}

37 À ce stade très en amont de la réflexion, il s'agissait de présenter à grands traits les versants sarrois et lorrain de la vallée et les dynamiques de reconversion qui les animent. Bien qu'ils se caractérisent par des états d'avancement variables (réalisés, en cours ou pressentis) ainsi que par des contraintes et intérêts spécifiques à l'échelle locale, il est important de souligner en quoi ces cinq projets gagneraient à s'intégrer dans un cadre cohérent, la vallée de la Rosselle revalorisée et réappropriée par ses habitants, dont la qualité des paysages, du patrimoine constituerait le dénominateur commun.

L'axe préservation-requalification du patrimoine industriel apporte en effet des réponses intéressantes aux attentes des communes en matière de qualité de vie, de cohésion urbaine et sociale, mais également de développement économique.

La fermeture programmée des sites, entre autre, pose aujourd'hui des questions cruciales de cohérence urbaine pour le devenir de ces territoires. Seules grandes surfaces en centre-ville, les terrains en friche présentent des opportunités uniques pour " recoudre la ville», et restaurer la continuité des espaces publics. Les projets d'intégration urbaine du site de Völklingen, le parc urbain du secteur St-Charles/StJoseph par exemple répondent aux attentes de la population pour des lieux de promenade et de culture, de rassemblement et de transition entre quartiers. La création de cheminements, de passerelles ouvre des perspectives visuelles sur des éléments de patrimoine oubliés et des pans de paysages ignorés. Un traitement raisonné des éléments bâtis et paysagers subsistants de l'activité industrielle devrait permettre une nouvelle lecture et une redécouverte des espaces quotidiens, pour les générations en place ou à venir. 
Par ailleurs, l'exploitation des carrières et des terrils a généré des paysages originaux, à mi-chemin entre nature et artifice, en renforçant le relief contrasté d'une topographie naturelle de côteaux. Peu à peu recolonisées par la végétation, ces nouvelles formes du relief ouvrent de saisissants points de vue paysagers, à valoriser dans le cadre d'opérations de reconquête. Cette morphologie atypique, ces ruptures visuelles et fonctionnelles des fronts de taille, en font des lieux peu propices à l'accueil d'activités économiques et plaident en faveur d'une réinsertion légère dans les trames urbaines. Là encore, la création d'espaces publics de promenade, de sports et de loisirs semble l'axe d'intervention le plus respectueux de l'identité des lieux et le plus pratique à mettre en œuvre, face aux contraintes physiques et réglementaires (affaissements, bassins à schlamms, réseaux,...)

41 La reconstitution d'ensembles à vocation touristique reste, quant à elle, un moyen privilégié pour promouvoir l'identité de cette région industrielle L'exemple de l'usine sidérurgique de Völklingen, du carreau Wendel, ou du projet de musée à Velsen ont montré l'intérêt d'une conservation sélective et d'une mise en circuit du patrimoine à des fins touristiques. Certes, les projets n'en sont qu'à leurs débuts, le Weltkulturerbe Völklinger Hütte est le seul pour l'instant à avoir su tirer son épingle du jeu, grâce notamment à la formidable impulsion que lui ont donné le classement au Patrimoine Mondial de l'Unesco et sa prise en charge financière directe par le Land.

La situation du carreau Wendel est toute autre. Sa lourde réhabilitation en cours depuis dix ans n'a pas suffi à drainer le public attendu sur le musée, qui peine encore à fonctionner faute de financements. Les tendances s'avèrent toutefois encourageantes. Les projets de liaisons cyclables avec Völklingen, la perspective de fonds européens et la nouvelle impulsion au partenariat avec le Weltkulturerbe Völklinger Hütte devraient lui donner l'élan nécessaire pour affirmer sa place de point d'ancrage de la culture et du tourisme industriel de la vallée.

43 Enfin sur le plan économique, la reconversion et le désenclavement d'anciens sites industriels et douaniers aux abords des villes de Sarrebruck et de Forbach constituent une voie intéressante pour les partenaires du projet, le Conseil Général de la Moselle, le Land de Sarre (Ministère de l'Economie), la ville de Sarrebruck et la Communauté d'Agglomération de Forbach, afin de concrétiser leur politique de rapprochement au niveau économique. Le site Eurozone permet d'illustrer la volonté de "gommer » une frontière et les obstacles qu'elle constitue, tout en restructurant des friches urbaines. Il s'agit d'une part de créer un site binational qui offre aux entreprises un accès direct et simplifié aux deux principaux marchés européens, à savoir la France et l'Allemagne ; d'autre part de mener une reconversion industrielle respectueuse du passé et de l'environnement ${ }^{5}$ en conservant le cachet architectural des sites classés des puits Simon, de l'ancienne douane et des logements des jardiniers du cimetière de Sarrebruck. Les nouvelles constructions qui seront appelées à occuper les différents espaces de l'Eurozone s'inscriront dans cette même démarche architecturale.

\section{B. Des potentialités indéniables}

L'étude de faisabilité du Parc de Développement de la Vallée de la Rosselle (figure 2) confirme le potentiel existant pour nourrir les thématiques d'interventions générales dégagées lors des premières réunions de travail (environnement et paysages, 
diversification de l'offre culturelle et touristique, recyclage urbain, innovation économique). Des enjeux forts se dégagent des sites étudiés :

- ils se trouvent à la jonction entre milieux naturels et humains, les plaçant à la fois dans un cadre paysager de qualité à préserver, et dans une situation stratégique pour le développement urbain ;

- ils présentent des vestiges de l'activité industrielle, de nature et de formes variées, dont la valeur historique s'affirme comme un levier potentiel pour en faire de nouveaux lieux de vie et d'attraction culturelle ;

- ils ont un rôle essentiel à jouer dans l'amélioration du cadre de vie, tant pour la qualité de vie urbaine au quotidien, que pour la relance de l'activité touristique et économique.

La vallée de la Rosselle bénéficie donc d'un support patrimonial original, qui en marque l'identité. Il mériterait d'être mis en valeur par une thématisation, qui fédérerait l'ensemble des projets de reconversion en cours et à mettre en œuvre. D'autre part, l'amorce d'une telle démarche d'aménagement, à la fois transversale et transfrontalière, présente une opportunité d'innovation opérationnelle. Il s'agit de concilier :

- l'originalité de chaque projet, lui assurant une gestion autonome et flexible : à l'échelle de la Lorraine et de la Sarre, une opportunité à saisir serait de rapprocher les attractions touristiques de la Rosselle d'autres particularités régionales non liées à l'industrie, telles que le Parc Archéologique Européen de Bliesbruck-Reinheim, le Château de Marlbrouck ou le méandre de la Sarre ;

- l'unité géographique et les complémentarités des problématiques abordées, permettant de donner un sens à l'ensemble, pour une nouvelle lecture du territoire. La piste des cheminements transfrontaliers tout le long de la Rosselle, si elle est encadrée par un cadre cohérent de promotion touristique pourrait servir de manière originale cette ambition.

\section{Une stratégie à élaborer}

Si le principe d'une coopération transfrontalière semble acquis, tant sur le plan politique que technique de nombreux aspects opérationnels sont encore à mettre en place dans le cadre en évolution que constitue l'Eurodistrict. Après échanges d'expériences, définition de thématiques générales de travail, et cadrage spatiotemporel, l'étude de faisabilité devrait arrêter un diagnostic commun (à noter la multitude d'études thématiques ou transversales lancées par les divers acteurs de part et d'autre de la frontière, qui handicapent la mise en place d'un projet de territoire commun et transversal), sensibiliser les différents acteurs concernés sur le sens et la portée de la démarche, enfin aboutir à l'adoption d'une vision réellement " partagée " $\mathrm{du}$ territoire, en s'entendant sur les objectifs à atteindre et le partage des responsabilités tant financière que technique, et une harmonisation des ordres de priorité. La création d'un GLCT (ou l'ouverture du GLCT de l'« Eurozone SarrebruckForbach Nord » à d'autres compétences) est l'une des dernières innovations juridiques européennes qui puissent à terme porter un tel projet.

\section{Conclusion}

À l'échelle élargie de la vallée de la Rosselle, la colonne verte de l'agglomération transfrontalière, les sites industriels et miniers se répondent et se complètent de part 
et d'autre de la frontière: chevalements, bâtiments techniques, carrières, terrils, massifs boisés, et cités ouvrières. Considérés non plus isolément, mais comme un ensemble visuel et fonctionnel cohérent, les héritages industriels et miniers prennent un nouveau sens, contribuant à la revalorisation de l'image de l'ensemble du bassin houiller.

Mais le haut niveau d'ambition du « Parc de Développement de la Vallée de la Rosselle » et de manière plus générale du projet d'agglomération transfrontalière SarrebruckMoselle Est, en fait aussi la faiblesse. Il suppose des conditions assez contraignantes : un soutien financier continu des pouvoirs publics, la mise au point d'une stratégie transfrontalière (partage clair des compétences et des responsabilités, harmonisation des objectifs et des ordres de priorité), et surtout un engagement total des partenaires. La volonté de coopérer, si elle est manifeste dans les discours, laisse en réalité la place à une logique d'acteurs figés dans le cadre national voir strictement local de leurs compétences, ce qui empêche bien souvent le passage des actions engagées à l'opérationnel.

Or la mise en place opérationnelle d'un projet comme le Parc de Développement de la Vallée de la Rosselle permettrait de maîtriser les coûts, de capter des crédits européens (Interreg III et IV en 2008), et de placer les opérations de remise en état des sites au cœur d'une valorisation d'ensemble du territoire, croisant les questions de valorisation patrimoniale et paysagère, de renouvellement urbain, de tertiarisation du tissu économique, dans une approche innovante et créative. La prise en compte globale de l'ensemble de ces enjeux est désormais la condition sine qua none de l'entrée de l'agglomération transfrontalière Sarrebruck-Moselle Est dans le jeu européen.

\section{BIBLIOGRAPHIE}

CLAUSSEN W. (1991). - Industriebrachenreaktivierung und regionale Strukturpolitik in der saarländischlothringischen Grenzregion: technologieorientierte Altlastensanierung versus Flächerekultivierung, Diplomarbeit Geographie, Universität Dortmund, 275 p.

СоOK A., HOURTE A.-C. (1996). - Patrimoine et culture industrielle en Lorraine, jalons sur une route de l'industre Saar-Lor-Lux, Metz, Éditions Serpenoise, Direction Régionale des Affaires Culturelles de Lorraine, CCSTI du fer et de la Métallurgie, Musée de l'Histoire du Fer, 224 p.

DAVIET S. (1990). - Le bassin houiller lorrain et son espace frontalier : l'évolution d'une région en voie de reconversion, Doctorat d'État présenté sous la direction de M.B.Barbier, Université d'Aix-Marseille II, Institut de Géographie, 367 p.

DÖRRENBÄCHER P. (2002). - « Die Entwicklung des Steinkohlebergbaus im Saarland und in Lothringen seit der Kohlekrise ». In : Herrmann H.W., Wynants P. - Dir., Acht Jahrhunderte Steinkohlenbergbau, Actes du Colloque international organisé aux facultés Universitaires NotreDame de la Paix par la Fondation Meuse-Moselle, Namur, 9-11 septembre 1999, Colloques MeuseMoselle, volume II, Namur, Facultés Universitaires notre-Dame de la paix, p 367-388. 
DÖRRENBÄCHER P. (1989). - « Entwicklung und räumliche Organisation der Saarbergwerke AG ». In : Soyez D. - Dir., Das Saarland, Beharrung und Wandel in einem peripheren Grenzraum, volume I, Saarbrücken, Geographisches Institut der Universität des Saarlandes, p 203-227.

FRECAUT R. - Dir (1983). - Géographie de la Lorraine, Nancy, Metz, Éditions Serpenoise et Presses Universitaires de Nancy, $463 \mathrm{p}$.

FRIEDMOND K. (2001). - Velsen, ein Industrekomplex im Warndt einst und heute, ehemalige Grube Velsen, Wasserwerk Lauterbachtal, ehemaliges Schwelwerk Velsen, Düngemittelfabrik AGRA, Betriebsgesellschaft Abfallverwertungsanlage Velsen, Völklingen-Ludweiler, Heimatkundlicher Verein Warndt e.V., 87 p.

HABY R. (1965). - Les Houillères de Lorraine et leur région, volume I, Paris, Éditions S.A.B.R.I, Ministère de l'Education Nationale, Direction de l'Enseignement Supérieur, Centre National de Recherche Scientifique, $521 \mathrm{p}$.

HAMMAN Ph. (2003). - « La coopération intercommunale transfrontalière : vers une nouvelle gouvernance locale en Europe ? L'exemple de l'association de communes Saar Moselle Avenir ». In : Politique et management public, vol 21, $\mathrm{N}^{\circ} 1$, Paris, Institut de management public, $56 \mathrm{p}$.

LONCAR N. (2003). - « Neues Rauschen im Rosseltal, eine Industrie-brache wandelt sich zur Natur aus zweiter hand - Bis 2005 soll die Umgestaltung fertig sein ». In :Saarbrücker Zeitung, quotidien, 22 septembre.

MATHIAS K., AMMON W. (1980). - Wirtschaftsgeographie des Saarlandes, Band 1, Saarbrücken, Buchverlag Saarbrücker Zeitung, p. 125-169, 287-355.

MOLL P. (2000). - « Industrie, Brache im Saarland, Zielsetzungen der Raumordnung », Mosella, tome XXV, $\mathrm{N}^{\circ} 1-2$, p. 143-148.

MORBACH F., BRÜCHER W. (1989). - « Steinkohlebergbau und leistungsgebundene Energiewirtschaft im Saarland unter dem Einfluss der Grenze ». In : Soyez D. - Dir., Das Saarland, Beharrung und Wandel in einem peripheren Grenzraum, volume I, Saarbrücken, Geographisches Institut der Universität des Saarlandes, 47 Deutschen Geographentages in Saarbrücken vom 2/7 Oktober 1989, p. 159-181.

SCHмiтT A. (1995). - Denkmäler saarländischer Industriekultur: Wegweiser zur Industriestrasse Saar-LorLux, Staatliches Konservatoramt Saarbrücken, Trier, Spee-Buchverlag, 180 p.

\section{Sites internet :}

- site de la Mission Opérationnelle Transfrontalière : www.espaces-transfrontaliers.org, rubrique Documents - Fonds Juridiques

- site de CdF : www.charbonnagesdefrance.de

- site de Saarprojekt GmbH : www.saarprojekt.de

- site de la GIU mbH : www.giu.de

- site du Konzern RAG AG : www.rag.de

- site des Deutsche Steinkohle AG : www.deutsche-steinkohle.de

- site du Gouvernement du Land de Sarre (Landesregierung) www.saarland.de 


\section{NOTES}

1. GIU/EPF Lorraine- Machbarkeitsstudie : das Zukunftsprogramm Rosseltal / Etude de Faisabilité : Parc de Développement de la Vallée de la Rosselle, sous maitrise d'ouvrage de la ville de Völklingen, présentée en février 2001 à Petite-Rosselle.

2. Traitement des espaces dégradés (TED) : politique régionale de traitement des friches industrielles, initiée dès le premier Contrat de Plan Etat-Région (1986-1990) et cofinancée par l'État, la Région, l'EPFL et l'Union Européenne (à partir du second Contrat de Plan État-Région).

3. TED : «Traitement des espaces dégradés ».

4. d'après le Code de l'Environnement du Land de Sarre, article 25, la vallée de la Rosselle est classée depuis 1988 « Landschaftsschutzgebiet », zone de protection naturelle.

5. Les demandes d'implantation des entreprises sont examinées en accordant une attention particulière aux aspects « environnement ", " qualités urbaines et architecturales » et « voisinage compatible ».

\section{RÉSUMÉS}

Les friches minières résultant du déclin de l'exploitation houillère dans le bassin sarro-lorrain sont longtemps restées en l'état, sans faire l'objet d'une valorisation. Avec le retrait entre 2006 et 2010 des charbonnages français et sarrois, une prise de conscience s'est engagée de la nécessité de reconvertir ces espaces marqués par la monoindustrie en espaces porteurs de développement. Une politique commune de valorisation des friches minières exploitant leur potentiel économique, urbain, culturel et paysager se dessine à l'échelle de l'agglomération transfrontalière Sarrebruck-Moselle Est.

Mining relicts developing with the decline of coal mining in the region Sarre and Lorraine were abandoned a long time without new use. With the shrinking of the coal industry in Lorraine and Sarre from 2006 to 2010 began the consciousness that it was necessary to remediate these abandoned sites in sites with future. A common concept for remediation of mining relicts and their economic, urban, cultural and landscape value arises in the cross-border urban region Saarbrücken-Moselle East.

Die Brachflächen, die mit dem Niedergang des Kohlenbergbaus im saarländischen-lothringischen Bergbaureviers entstanden, blieben lange ungenutzt. Mit dem Rückbau der saarländischen und französischen Bergbaugesellschaften von 2006 bis 2010 entstand ein Bewusstwerden, dass es nötig war, diese durch Monindustrie geprägten Brachen in Entwicklungsflächen umzustrukturieren. Eine gemeinsame Politik zur Aufwertung der Brachflächen des Bergbaus zeichnet sich im Rahmen der grenzübergreifenden Agglomeration Saarbrücken-Moselle Ost.

\section{INDEX}

Mots-clés : friches minières, réhabilitation, coopération transfrontalière

Schlüsselwörter : Bergbaubrachflächen, Renaturierung, grenzübergreifende Zusammenarbeit

Keywords : mining relicts, remediation, cross-border cooperation 


\section{AUTEUR}

\section{JULIETTE RIPP}

Centre d'Études et de Recherches sur les Paysages (CERPA) - Université de Nancy 2 - 23, bd Albert $1^{\text {er }} 54015$ Nancy CEDEX 